Yuri Takahashi, Mio Sakuma, Hiroki Murayama and Takeshi Morimoto*

\title{
Effect of baseline renal and hepatic function on the incidence of adverse drug events: the Japan Adverse Drug Events study
}

https://doi.org/10.1515/dmpt-2018-0018

Received June 28, 2018; accepted October 3, 2018; previously published online November 10, 2018

\section{Abstract}

Background: The impact of renal and hepatic dysfunction on the morbidity and mortality of inpatients with adverse drug events (ADEs) is uncertain in daily clinical practice. The objective of this study was to investigate the effect of renal and hepatic function on ADEs and inpatients' morbidity and mortality.

Methods: The Japan Adverse Drug Events (JADE) study was a prospective cohort study carried out at three tertiary-care teaching hospitals in Japan. Participants were consecutive inpatients $(n=3459)$ aged 15 years or older. We evaluated the effect of renal and hepatic function on the occurrence of ADEs, and assessed how they affected length of hospital stay (LOS) and in-hospital mortality. We used the estimated glomerular filtration rate to quantify renal function and categorized patients into three groups (normal, $\geq 60 \mathrm{~mL} / \mathrm{min} / 1.73 \mathrm{~mm}$; moderate, $\geq 30$ and $<60 \mathrm{~mL} / \mathrm{min} / 1.73 \mathrm{~mm}$; severe, $<30 \mathrm{~mL} / \mathrm{min} / 1.73 \mathrm{~mm}$ ). We defined patients as having hepatic dysfunction when at least one data point (total bilirubin, aspartate aminotransferase, alanine aminotransferase, or gamma glutamyltransferase) was beyond a cutoff value.

Results: We analyzed the laboratory data of 2508 patients. There was a significant difference in the occurrence of ADEs among the three GFR categories (normal, $20 \%$; moderate, $26 \%$; severe, $22 \%$; $\mathrm{p}=0.02$ ). More ADEs occurred in patients with hepatic dysfunction (25\% vs. $20 \%, p=0.01)$. LOS was significantly longer in those with ADEs stratified either by renal or by hepatic dysfunction

\footnotetext{
*Corresponding author: Takeshi Morimoto, MD, PhD, MPH, Department of Clinical Epidemiology, Hyogo College of Medicine, Nishinomiya, Hyogo 663-8501, Japan, Phone: +81-798-45-6879, Fax: +81-798-45-6920, E-mail: t-morimoto@umin.net. http://orcid.org/0000-0002-6844-739X Yuri Takahashi, Mio Sakuma and Hiroki Murayama: Department of Clinical Epidemiology, Hyogo College of Medicine, Nishinomiya, Hyogo, Japan
}

$(\mathrm{p}<0.0001)$. ADEs were independently associated with inhospital mortality, adjusting for renal and hepatic function $(\mathrm{p}<0.0001)$.

Conclusions: Inpatients' organ dysfunction increased ADEs, and ADEs were associated with both LOS and inhospital mortality independently, irrespective of renal and hepatic function.

Keywords: adverse drug events; hepatic function; JADE study; patient safety; renal function.

\section{Introduction}

Adverse drug events (ADEs) are injuries from medication usage $[1,2]$ and are a cause of morbidity, mortality, and hospitalization $[1,3]$. Many inpatients with acute or chronic diseases need to take multiple medications for treatment. Because all medications pass through the processes of absorption, distribution, metabolism, and excretion (ADME), declines in ADME functions of organs with aging, injury, and disease influence the safety of medications [4-6]. In daily clinical practice, multi-medication therapies are used for patients with comorbidities or complications. However, we know little about how many ADEs occur in such patients in daily clinical practice, including patients with renal or hepatic dysfunction, except what we learn from clinical trials. Furthermore, the influence of ADEs on in-hospital mortality or on the length of hospital stay (LOS) of patients with organ dysfunction has not been reported.

In our previous Japan Adverse Drug Events (JADE) study, we evaluated the incidence of ADEs among 3459 hospitalized patients and found that 726 patients had 1010 ADEs during hospitalization, and $6.5 \%$ of these ADEs were life-threatening [7]. We are interested in how renal and hepatic dysfunction affects the morbidity and mortality of patients with ADEs in daily clinical practice. Therefore, we investigated how inpatients' renal and hepatic function was related to the occurrence of ADEs. We also investigated the influence of ADEs on in-hospital mortality and on LOS, taking renal and hepatic dysfunction into account. 


\section{Materials and methods}

\section{Study design and patient population}

The JADE study was a prospective cohort study of 3459 patients aged 15 years or older who were admitted to three tertiary-care hospitals in Japan from January to June 2004. These patients were admitted to 15 medical and surgical wards and three intensive care units in these hospitals [7]. Patients were followed until transfer, discharge, or death.

\section{Ethics approval and consent to participants}

The study protocol complied with the Declaration of Helsinki and the guidelines for epidemiological studies issued by the Ministry of Health, Labour, and Welfare in Japan. The institutional review boards of the three participating hospitals (St. Luke's International Hospital, Rakuwakai Otowa Hospital, and Aso Iizuka Hospital) and the Ethics Committee of the Kyoto University Graduate School of Medicine approved the study (E-15). Informed consent was waived because all data were collected in daily clinical practice. This waiver was approved by the institutional review boards.

\section{Data collection and review process}

The data collection method was based on that described in a previous report [2]. An ADE was defined as any unintended injury related to medication usage, regardless of existing errors $[2,8]$. In the first step, trained research assistants reviewed all practice data (such as medical charts, laboratories, prescription data, incident reports, and prescription queries). They also collected the patient characteristics. Comorbidity in the patients was quantified using the Charlson Comorbidity Index [9].

In the second step, two independent physician reviewers evaluated and classified all data collected by the research assistants as either ADEs or exclusion.

Interrater reliabilities were assessed using $\kappa$ statistics. The $\kappa$ scores regarding presence of an ADE between reviewers were 0.75 (ADE vs. potential ADE or exclude) and 0.77 (exclude vs. ADE or potential ADE). The $\kappa$ for preventability was 0.86 (preventable vs. nonpreventable), whereas $\kappa$ scores for severity were 0.31 (life-threatening vs. serious or significant) and 0.64 (significant vs. serious or life-threatening) [1].

\section{Renal and hepatic dysfunction}

Laboratory data were collected on admission. We calculated the estimated glomerular filtration rate (eGFR) from serum creatinine on admission and divided the patients into the following three categories according to the Japanese CKD guideline [10]. We considered those with eGFR $\geq 60 \mathrm{~mL} / \mathrm{min} / 1.73 \mathrm{~mm}$ as having normal renal function, those with $\geq 30$ and $<60 \mathrm{~mL} / \mathrm{min} / 1.73 \mathrm{~mm}$ as having moderate dysfunction, and those with $<30 \mathrm{~mL} / \mathrm{min} / 1.73 \mathrm{~mm}$ as having severe dysfunction.
We used total bilirubin, aspartate aminotransferase (AST), alanine aminotransferase (ALT), and gamma glutamyltransferase (GGTP) as measurements of hepatic function. We defined hepatic dysfunction as having at least one of the four laboratory data points of the hepatic function data beyond a cutoff value. Cutoff values were set from the classification criteria for the seriousness of adverse drug reactions to medications, developed by the Ministry of Health and Welfare in Japan [11]: total bilirubin $\geq 3.0 \mathrm{mg} / \mathrm{dL}$, AST $\geq 100 \mathrm{IU} / \mathrm{L}$, ALT $\geq 100 \mathrm{IU} / \mathrm{L}, \mathrm{GGTP} \geq 105 \mathrm{IU} / \mathrm{L}$ (male), and GGTP $\geq 45 \mathrm{IU} / \mathrm{L}$ (female).

\section{Statistical analyses}

Continuous variables are presented as mean \pm standard deviation (SD) or median (interquartile range), and categorical variables are shown as numbers and percentages. Relationships between patient's demographic data and ADEs were assessed using the Wilcoxon rank-sum test when the data were continuous and the $\chi^{2}$ test when the demographic data were categorical. We compared the occurrence of ADEs between patients with and without renal dysfunction, and patients with and without hepatic dysfunction. We compared the occurrence of ADEs between patients with less than five medications on admission and those with five or more medications on admission, and counterpart stratified the test by renal and hepatic dysfunction. We divided the number of medications used into two categories $(<5$ and $\geq 5)$ based on our previous report from the JADE study [7].

We compared LOS and in-hospital mortality between those with ADEs and those without ADEs, stratified by renal or hepatic dysfunction. We also conducted sensitivity analyses excluding the patients who died within 2 days after admission because such patients showed renal or hepatic dysfunction on admission and their abnormal laboratory data and poor prognosis were not associated with ADEs or longer LOS. We finally developed a logistic regression model to assess the effect of renal and hepatic dysfunction on in-hospital mortality, adjusting for age, presence of ADEs, and the number of medications used on admission in the sensitivity analysis cohort. Two-tailed $p$-values $<0.05$ were considered statistically significant.

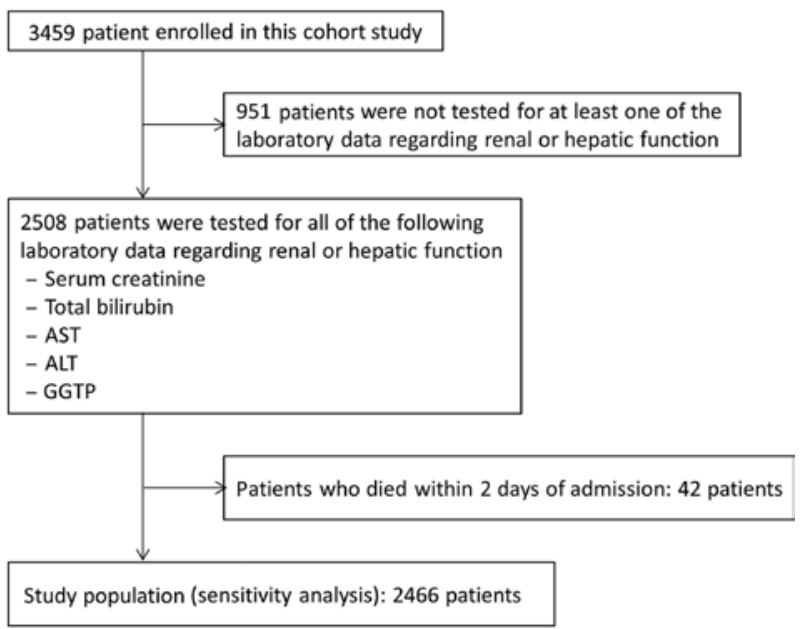

Figure 1: Flowchart of patients. 
Table 1: Characteristics and demographics of patients on admission.

\begin{tabular}{|c|c|c|c|c|}
\hline Characteristics & Total $(n=2508)$ & With ADEs $(n=546)$ & Without ADEs $(n=1962)$ & p-Value \\
\hline Age, years, mean $\pm S D$ & $66.1 \pm 16.9$ & $70.3 \pm 14.1$ & $64.9 \pm 17.4$ & $<0.0001$ \\
\hline Men, n (\%) & $1441(58)$ & $309(57)$ & $1132(58)$ & 0.6 \\
\hline Body mass index, mean \pm SD & $22.3 \pm 4.0$ & $21.4 \pm 4.0$ & $22.5 \pm 3.9$ & $<0.0001$ \\
\hline Wards, n (\%) & & & & 0.001 \\
\hline Surgical & $1132(45)$ & $261(48)$ & $871(44)$ & \\
\hline Medical & $1022(41)$ & $233(43)$ & $789(40)$ & \\
\hline ICUs & $354(14)$ & $52(10)$ & $302(15)$ & \\
\hline Charlson index score, median ( $25 \%-75 \%)$ & $3(1-5)$ & $3(1-5)$ & $2(1-5)$ & $<0.0001$ \\
\hline $\mathrm{SBP}(\mathrm{mmHg})$, mean $\pm \mathrm{SD}$ & $131.8 \pm 24.3$ & $133.0 \pm 25.9$ & $131.4 \pm 23.9$ & 0.4 \\
\hline $\mathrm{DBP}(\mathrm{mmHg})$, mean $\pm \mathrm{SD}$ & $73.4 \pm 14.1$ & $73.7 \pm 13.6$ & $73.3 \pm 14.2$ & 0.9 \\
\hline Renal function, $\mathrm{n}(\%)$ & & & & 0.01 \\
\hline Normal renal function & $1664(66)$ & $336(62)$ & $1328(68)$ & \\
\hline Moderate renal dysfunction & $584(23)$ & $152(28)$ & $432(22)$ & \\
\hline Severe renal dysfunction & $260(10)$ & $58(11)$ & $202(10)$ & \\
\hline Hepatic function, n (\%) & & & & 0.01 \\
\hline Normal hepatic function & $1716(68)$ & $349(64)$ & $1367(70)$ & \\
\hline Hepatic dysfunction & $792(32)$ & $197(36)$ & $595(30)$ & \\
\hline \multicolumn{5}{|l|}{ Drug, n (\%) } \\
\hline Antibiotics & $797(32)$ & $188(34)$ & $609(31)$ & 0.13 \\
\hline Antitumor agents & $63(3)$ & $12(2)$ & $51(3)$ & 0.59 \\
\hline Diuretics & $391(16)$ & $81(15)$ & $310(16)$ & 0.58 \\
\hline Antihypertensive & $661(26)$ & $148(27)$ & $513(26)$ & 0.65 \\
\hline Antiarrhythmic & $57(2)$ & $12(2)$ & $45(2)$ & 0.89 \\
\hline Cardiovascular & $466(19)$ & $98(18)$ & $368(19)$ & 0.67 \\
\hline Anticoagulants & $279(11)$ & $58(11)$ & $221(11)$ & 0.67 \\
\hline Dyslipidemic agents & $142(6)$ & $30(5)$ & $112(6)$ & 0.85 \\
\hline Antidiabetics & $287(11)$ & $67(12)$ & $220(11)$ & 0.49 \\
\hline Antiasthmatics & $96(4)$ & $21(4)$ & $75(4)$ & 0.98 \\
\hline Peptic ulcer drugs & 890 (35) & $202(37)$ & $688(35)$ & 0.40 \\
\hline Laxatives & $427(17)$ & $110(20)$ & $317(16)$ & 0.028 \\
\hline Antidepressants & $30(1)$ & $7(1)$ & $23(1)$ & 0.83 \\
\hline Sedatives & $955(38)$ & $225(41)$ & $730(37)$ & 0.087 \\
\hline Antipsychotics & $149(6)$ & $44(8)$ & $105(5)$ & 0.018 \\
\hline Antiseizure & $69(3)$ & $19(3)$ & $50(3)$ & 0.24 \\
\hline Anti-Parkinson's drugs & $38(2)$ & $10(2)$ & $28(1)$ & 0.49 \\
\hline Muscle relaxant & $70(3)$ & $17(3)$ & $53(3)$ & 0.61 \\
\hline NSAIDs & $569(23)$ & $151(28)$ & $418(21)$ & 0.0017 \\
\hline Other analgesics & $666(27)$ & $156(29)$ & $510(26)$ & 0.23 \\
\hline Corticosteroids & $145(6)$ & $41(8)$ & $104(5)$ & 0.051 \\
\hline Antihistamines & $83(3)$ & $20(4)$ & $63(3)$ & 0.60 \\
\hline Electrolytes or fluids & $1338(53)$ & $284(52)$ & $1054(54)$ & 0.48 \\
\hline Experimental drugs & $1(0.04)$ & $1(0.2)$ & $0(0)$ & 0.058 \\
\hline Others & $1547(62)$ & $342(63)$ & $1205(61)$ & 0.60 \\
\hline
\end{tabular}

ICUs, intensive care units; SBP, systolic blood pressure; DBP, diastolic blood pressure; NSAIDs, nonsteroidal antiinflammatory drugs.

We carried out all analyses using the JMP 11.2 software (SAS Institute Inc., Cary, NC, USA).

\section{Results}

Laboratory data of both renal and hepatic function were available for 2508 of the 3459 patients enrolled (Figure 1).
After excluding 42 patients who died within 2 days who had no ADE, the data of 2466 patients were used in the sensitivity analysis.

Among the 2508 patients, 546 had ADEs. The mean age was significantly higher in patients with ADEs than in those without ( 70.3 vs. 64.9 years, $\mathrm{p}<0.0001)$. The mean Charlson index score was also significantly higher in patients with ADEs (3 vs. 2, p $<0.0001$ ), whereas 

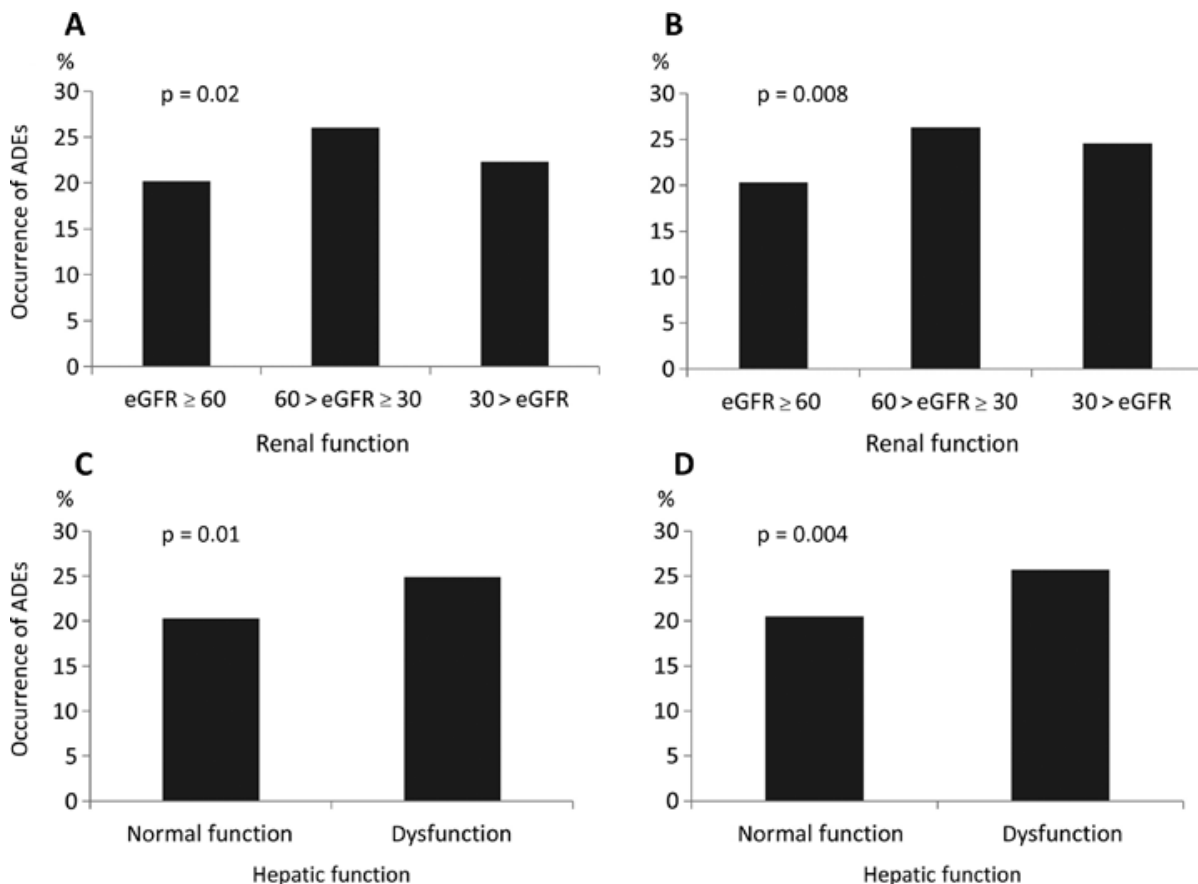

Figure 2: Effect of organ function on ADEs.

(A) The occurrence of ADEs in patients stratified by eGFR category $(<30 ; \geq 30$ and $<60 ; \geq 60 \mathrm{~mL} / \mathrm{min} / 1.73 \mathrm{~mm})$. (B) Sensitivity analysis of the occurrence of ADEs in patients in the three eGFR categories. (C) The occurrence of ADEs in patients with normal hepatic function and hepatic dysfunction. (D) Sensitivity analysis of the occurrence of ADEs in patients with normal hepatic function and hepatic function abnormalities.

body mass index was significantly lower (21.4 vs. 22.5, $\mathrm{p}<0.0001)$. The categories of renal and hepatic function were also significantly different between the two groups (Table 1).

\section{Effect of renal and hepatic dysfunction on ADEs}

The occurrence of ADEs was significantly different among eGFR categories [normal function, $20 \%(n=336)$; moderate dysfunction, $26 \%(\mathrm{n}=152)$; and severe dysfunction, $22 \%(n=58) ; p=0.02$ ] (Figure 2A). The occurrence of ADEs was also significantly different between hepatic function categories [normal function, 20\% $(\mathrm{n}=349)$; dysfunction, $25 \%(\mathrm{n}=197) ; \mathrm{p}=0.01$ ] (Figure 2C). The sensitivity analyses, excluding patients who died within 2 days, showed similar results [normal renal function, $20 \%,(\mathrm{n}=336)$; moderate renal dysfunction, $26 \%(\mathrm{n}=152)$; and severe renal dysfunction, $25 \%(\mathrm{n}=58) ; \mathrm{p}=0.008$; and normal hepatic function, 20\% ( $\mathrm{n}=349)$; dysfunction, $26 \%$ $(\mathrm{n}=197) ; \mathrm{p}=0.004]$ (Figure 2B and D). Among the 792 patients with hepatic dysfunction, the occurrence of ADEs was higher in the elderly [ $\geq 65$ years old, $28 \%(\mathrm{n}=131)$ vs. $20 \%(\mathrm{n}=66) ; \mathrm{p}=0.007]$.

\section{Effect of number of medications used on ADEs}

Among those with normal renal function, ADE occurrence was significantly higher in patients to whom five or more medications were prescribed on admission than in those who were prescribed less than five $[25 \%(n=143)$ vs. $18 \%$ ( $n=193), p=0.0005$ ] (Figure 3A). However, these effects were not observed among those with moderate or severe renal dysfunction [moderate dysfunction, $25 \%(\mathrm{n}=60)$ vs. $27 \%(\mathrm{n}=92), \mathrm{p}=0.5$; severe dysfunction, $23 \%(\mathrm{n}=35)$ vs. $22 \%(\mathrm{n}=23), \mathrm{p}=0.8]$. Among those with normal hepatic function, ADE occurrence was also significantly higher in patients to whom five or more medications were prescribed on admission than in those who were prescribed less than five [24\% $(n=159)$ vs. $18 \%(n=190), p=0.007]$ (Figure 3C). This effect was also not observed among those with hepatic dysfunction. The results of the sensitivity analyses were similar [normal renal function, 25\% $(\mathrm{n}=143)$ vs. $18 \%(\mathrm{n}=193), \mathrm{p}=0.0005$; moderate renal dysfunction, $25 \%(\mathrm{n}=60)$ vs. $27 \%(\mathrm{n}=92), \mathrm{p}=0.5$; severe renal dysfunction, $24 \%(n=35)$ vs. $25 \%(n=23), p=0.9$; and normal hepatic function, $24 \%(\mathrm{n}=159)$ vs. $18 \%$ $(\mathrm{n}=190), \mathrm{p}=0.008$; hepatic dysfunction, $27 \%(\mathrm{n}=79)$ vs. $25 \%(n=118), p=0.4]$ (Figure 3B and D). 
A
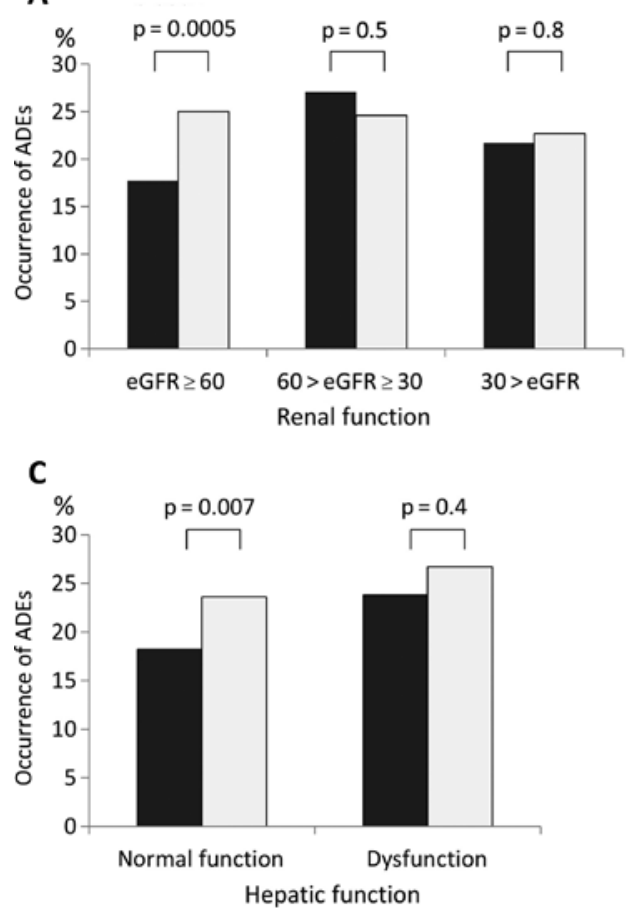

B

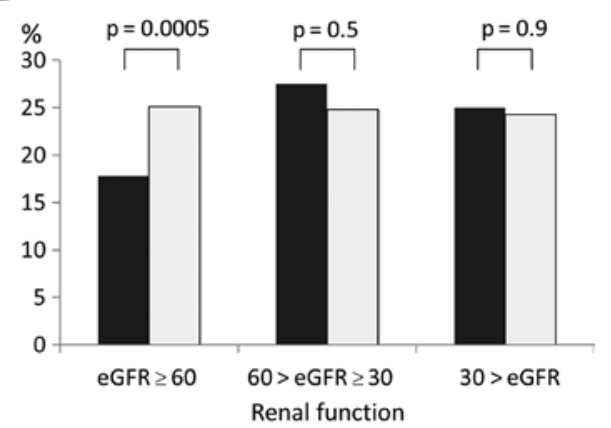

D

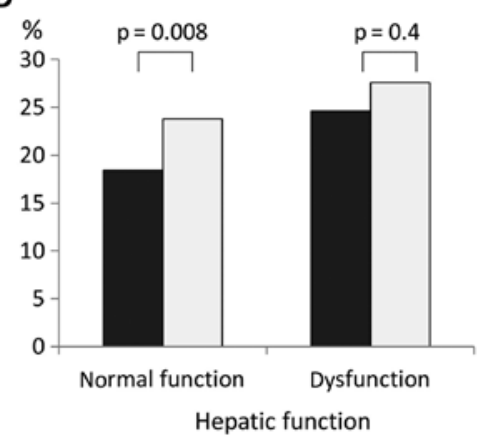

$$
\begin{aligned}
& \square 4 \geq \\
& \square \geq 5 \\
& \text { (Medications on admission) }
\end{aligned}
$$

Figure 3: Effect of the number of medications used on ADEs, stratified by organ function.

(A) The occurrence of ADEs in patients in the three eGFR categories $(<30 ; \geq 30$ and $<60 ; \geq 60 \mathrm{~mL} / \mathrm{min} / 1.73 \mathrm{~mm})$, stratified by the number of medications used. (B) Sensitivity analysis of the occurrence of ADEs in patient in the three eGFR categories, stratified by the number of medications used. (C)The occurrence of ADEs in patients with normal hepatic function and hepatic dysfunction, stratified by the number of medications used. (D) Sensitivity analysis of the occurrence of ADEs in patients with normal hepatic function and hepatic dysfunction, stratified by the number of medications used. Black bars, the number of medications used is four or less; white bars, the number of medications used is five or more.

\section{Effect of ADEs on LOS}

The median LOS of patients with ADEs was longer than that of patients without ADEs, among those with normal renal function ( 20 vs. 7 days, $\mathrm{p}<0.0001$ ) and those with renal dysfunction (moderate renal dysfunction, 26 vs. 9 days, $\mathrm{p}<0.0001$; severe renal dysfunction, 22 vs. 6 days, $\mathrm{p}<0.0001)$. It was also longer among those with normal hepatic function ( 21 vs. 7 days, $\mathrm{p}<0.0001)$ and those with hepatic dysfunction ( 23 vs. 8 days, $p<0.0001$ ). The results of the sensitivity analyses were similar.

\section{Effect of ADEs on in-hospital mortality}

In-hospital mortality was higher in patients with ADEs than in patients without ADEs, among patients with normal renal function and moderate renal dysfunction [normal renal function, $13.7 \%(\mathrm{n}=46)$ vs. 3.9\% $(\mathrm{n}=52)$, $\mathrm{p}<0.0001$; moderate renal dysfunction, $15.1 \%(\mathrm{n}=23)$ vs. $8.3 \%(n=36), p=0.02$ ] (Figure 4A). However, these effects were not observed among those with severe renal dysfunction [24.1 $(n=14)$ vs. $20.8(n=42), p=0.6$ ]. In the sensitivity analysis, in-hospital mortality showed the same tendencies in the normal renal function and moderate renal dysfunction groups. However, in this analysis, inhospital mortality was also higher in patients with ADEs among those with severe renal dysfunction [24.1 $(n=14)$ vs. 10.1 ( $n=18), p=0.01$ ] (Figure 4B). Similarly, in-hospital mortality was higher in patients with ADEs among those with normal hepatic function [13.2 $(n=46)$ vs. $4.8(n=65)$, $\mathrm{p}<0.0001]$ and hepatic dysfunction $[18.8 \%(\mathrm{n}=37)$ vs. $10.9 \%(n=65), p=0.006$ ] (Figure 4C). The hepatic function results of the sensitivity analyses were similar (Figure 4D). 
A

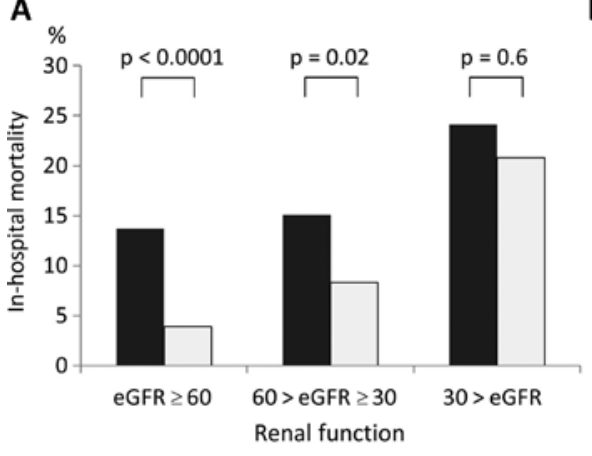

B \%

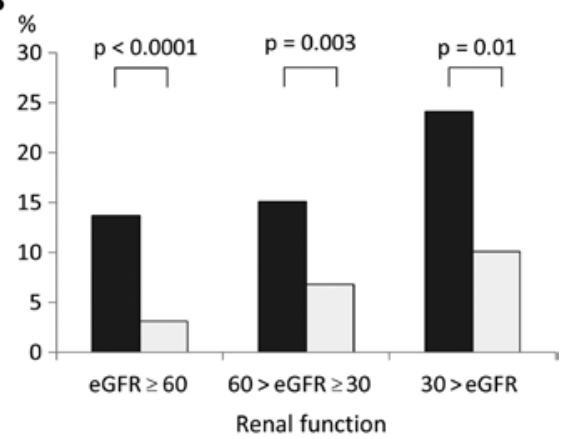

C

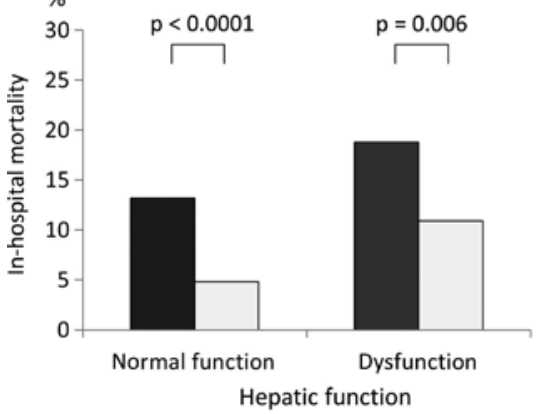

D \%

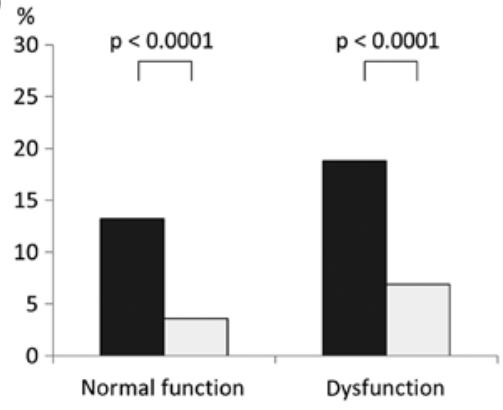

Hepatic function

\section{- $\mathrm{ADE}$}

$\square$ non-ADE

Figure 4: Effect of ADEs on in-hospital mortality, stratified by organ functions.

(A) In-hospital mortality in patients in the three eGFR categories $(<30 ; \geq 30$ and $<60 ; \geq 60 \mathrm{~mL} / \mathrm{min} / 1.73 \mathrm{~mm})$, stratified by ADE occurrence. (B) Sensitivity analysis of in-hospital mortality in patients in each eGFR category, stratified by ADE occurrence. (C) In-hospital mortality in patients with normal hepatic function and hepatic dysfunction, stratified by ADE occurrence. (D) Sensitivity analysis of in-hospital mortality in patients with normal hepatic function and hepatic dysfunction, stratified by ADE occurrence.

\section{Effect of renal and hepatic dysfunction on in-hospital mortality}

The multivariate logistic regression model showed moderate and severe renal dysfunction were significantly associated with in-hospital mortality [odds ratio (OR) of moderate relative to normal, 1.49 (95\% confidence interval, CI, 1.04-2.12); OR of severe relative to normal, 4.12 (95\% CI, 2.81-6.02)]. Hepatic dysfunction was also significantly associated with in-hospital mortality (OR, 2.08; 95\% CI, 1.55-2.79). The occurrence of ADEs was also independently associated with in-hospital mortality, adjusting

Table 2: Effect of renal and hepatic dysfunction on in-hospital mortality by univariate and multivariate analysis.

\begin{tabular}{|c|c|c|c|c|}
\hline \multirow[t]{3}{*}{ Variables } & \multicolumn{2}{|l|}{ Univariate } & \multicolumn{2}{|l|}{ Multivariate } \\
\hline & \multicolumn{2}{|l|}{ OR } & \multicolumn{2}{|l|}{ OR } \\
\hline & $(95 \% \mathrm{Cl})$ & p-Value & $(95 \% \mathrm{Cl})$ & p-Value \\
\hline ADEs & $2.53(1.88-3.39)$ & $<0.0001$ & $2.36(1.74-3.20)$ & $<0.0001$ \\
\hline Age $\geq 65$ years & $2.05(1.48-2.83)$ & $<0.0001$ & $1.67(1.19-2.37)$ & 0.0029 \\
\hline \multicolumn{5}{|c|}{ Renal dysfunction (eGFR, mL/min/1.73 mm) } \\
\hline$\geq 60$ & 1 (reference) & & 1 (reference) & \\
\hline$\geq 30$ and $<60$ & $1.29(0.94-1.77)$ & 0.12 & $1.49(1.04-2.12)$ & 0.029 \\
\hline$<30$ & $3.66(2.61-5.12)$ & $<0.0001$ & $4.12(2.81-6.02)$ & $<0.0001$ \\
\hline Hepatic dysfunction & $2.13(1.61-2.84)$ & $<0.0001$ & $2.08(1.55-2.79)$ & $<0.0001$ \\
\hline No. of medications $\geq 5$ & $1.34(0.78-1.38)$ & 0.81 & $0.83(0.61-1.12)$ & 0.23 \\
\hline
\end{tabular}


for renal and hepatic dysfunction (OR, 2.36; 95\% CI, 1.743.20) (Table 2).

\section{Discussion}

We found that approximately $30 \%$ of unselected inpatients in acute-care hospitals had renal or hepatic dysfunction, and that the risk of ADEs in such patients was significantly higher than in patients with normal organ function. We also found that the variables associated with increased occurrence of ADEs, such as being elderly, having renal dysfunction, and having hepatic dysfunction, were also independently associated with in-hospital mortality.

However, the occurrence of ADEs with severe renal dysfunction was smaller than the occurrence with moderate renal dysfunction. In-hospital mortality was significantly associated with renal dysfunction. Therefore, more patients with renal dysfunction would die before experiencing ADEs during the hospital stay. Indeed, the occurrence of ADEs with severe renal dysfunction increased when patients who died within 2 days were excluded.

Our findings were consistent with those from a previous study, which showed that a substantial proportion $(7.5 \%-10.4 \%)$ of patients admitted to acute-care hospitals experienced ADEs, with some of them being fatal [12].

Prevention of ADEs is expected to improve the prognosis of patients. In the United States, ADEs contribute to as many as 140,000 deaths annually, occurring in about 1 of 16 hospitalized patients. An estimated $28 \%$ to $56 \%$ of ADEs are preventable, and most preventable ADEs are due to errors during prescription [12]. A UK study showed that $12 \%$ of all primary-care patients may be affected by a prescribing or monitoring error over the course of a year, increasing to $38 \%$ in those aged 75 years and older and $30 \%$ in patients receiving five or more drugs during a 12-month period. Overall, about $5 \%$ of prescriptions are believed to have prescribing errors [13]. The WHO has provided a list of 10 key actions that are likely to have the most impact on improving safety in primary care, and one of them is to focus on those at a higher risk of safety incidents [14]. Our study showed that ADEs occurred significantly more in patients with organ dysfunction. Thus, intensive monitoring of such patients would contribute to reducing the incidence of ADEs, morbidity, and mortality.

In patients with normal renal or hepatic function in this study, the occurrence of ADEs increased when the number of medications increased. However, this tendency was not observed in patients with renal or hepatic dysfunction. Field et al. [15] reported that the risk of ADEs increased when the number of regularly scheduled medications was more than five in patients with normal renal and hepatic function, and our results were consistent with this report. Generally, drugs and their metabolites are excreted in the urine after polarization by a drug metabolism process in the liver [5]. If patients have hepatic or renal dysfunction, then this metabolism or excretion process has deteriorated. The relationship between the number of medications and occurrence of ADEs was not observed in patients with renal or hepatic dysfunction because of the decreased metabolism and excretion function. Even with only a few drugs administered, the blood concentration of these drugs or their metabolites increases causing enhanced drug sensitivity in patients with renal or hepatic dysfunction [16-22]. We suggested that the risk of ADEs depends on the number of medications in patients with normal metabolism, whereas the risk of ADEs was high even with a small number of medications in patients with decreased metabolism.

The efficiency of renal and hepatic function changes with age [23-25], and mean age on admission was 70 years in patients with ADEs in our study. Budnitz et al. [26] reported in a US study that there were an estimated 99,628 emergent hospitalizations for ADEs in adults aged 65 years or older each year from 2007 to 2009. Nearly half of these hospitalizations were reported for adults aged 80 years or older, and nearly two thirds of those were due to unintentional overdoses. In the same study, two thirds of the ADEs involved drugs such as warfarin, insulin, oral antiplatelet agents, and oral hypoglycemic agents. More caution during prescription is needed because many medications could cause renal or hepatic dysfunction [27-31]. In contrast, Dreischulte et al. [32] reported in a Scotland study that a complex intervention combining professional education, informatics, and financial incentives reduced the rate of high-risk prescribing of antiplatelet medications and nonsteroidal antiinflammatory drugs. The proper use and dose of medications are more important for elderly patients with renal or hepatic dysfunction because our results indicated that being elderly and having renal or hepatic dysfunction and ADEs were independently associated with in-hospital mortality. Furthermore, monitoring of renal and hepatic function should be approached with more attention in cases of multiple medication therapy.

Several limitations must be addressed regarding this study. First, the number of all medications were not available during the hospitalization. The changes in laboratory data after admission were also not assessed. Although the 
primary purpose of this study was to estimate the risk of ADEs and in-hospital mortality based on the renal and hepatic functions on admission, the changes in medication use and laboratory data could be incorporated to risk stratification. Second, we also did not assess the established indicators of hepatic function, which are widely used for the prognosis of liver disease, such as the ChildPugh score [33]. Therefore, the effect of renal and hepatic function on the occurrence of ADEs might be different if we used different indicators. Third, we did not consider pharmacogenomics or pharmacokinetic/pharmacodynamic studies to estimate the risks of ADEs in this study because such tests were not used in all patients in daily practice. We focused on the risk of ADEs based on renal and hepatic function, which are measured in all patients on admission. However, the risk stratification ability should be improved if we used such tests in the future. Finally, the JADE study only enrolled Japanese patients, and the study was conducted in 2004, with data that seem relatively old. To generalize our results globally, we need to study the effect of renal and hepatic function on the occurrence of ADEs in other countries to evaluate their effects among different ethnic groups and also in different healthcare systems, which can affect decision-making by healthcare professionals. However, as the medications used in this study have not been changed for decades, our findings and their clinical implication should be considered relevant in the present.

\section{Conclusions}

We found that renal and hepatic dysfunction increased the occurrence of ADEs, and that ADEs were associated with longer LOS and higher mortality in patients with both normal and decreased renal or hepatic function. Therefore, the appropriate and careful use of medication should be promoted, especially in patients with renal or hepatic dysfunction. Systems to confirm the necessity of organ function tests depending on the medications that a patient is taking, and to increase the timely identification and interception of ADEs according to renal or hepatic function, should be implemented to ensure the safer use of medication.

Acknowledgments: The JADE study for adult inpatients was conducted by the following investigators: Kunihiko Matsui, MD, MPH; Nobuo Kuramoto, MD; Jinichi Toshiro, MD; Junji Murakami, MD; Tsuguya Fukui, MD, MPH; Mayuko Saito, MD, MPH; Atsushi Hiraide, MD; and David
W. Bates, MD, MSc. We are also indebted to Ms. Makiko Ohtorii, Ms. Ai Mizutani, Ms. Mika Sakai, Ms. Izumi Miki, Ms. Kimiko Sakamoto, Ms. Eri Miyake, Ms. Takako Yamaguchi, Ms. Yoko Oe, Ms. Kyoko Sakaguchi, Ms. Kumiko Matsunaga, Ms. Yoko Ishida, Ms. Kiyoko Hongo, Ms. Masae Otani, Ms. Yasuko Ito, Ms. Ayumi Samejima, and Ms. Shinobu Tanaka for their data collection and management.

Author contributions: YT, MS, and TM planned this study. YT, HM, MS, and TM conducted the analysis, and wrote the draft and the final manuscript. All the authors have accepted responsibility for the entire content of this submitted manuscript and approved submission.

Research funding: This work was supported by JSPS KAKENHI (grant nos. JP17689022, JP21659130, JP22390103, JP23659256, JP26293159, JP18H03032), the grants from the Ministry of Health, Labour and Welfare of Japan (H26-Iryo-012, H28-ICT-004), the grants from the Pfizer Health Research Foundation, and the Uehara Memorial Foundation.

Employment or leadership: YT is an employee of Novartis Pharma KK. HM is an employee of Novartis Pharma KK. MS and TM have declared that they do not have any conflicts of interest pertaining to this manuscript.

Honorarium: None declared.

Competing interests: The funding organization(s) played no role in the study design; in the collection, analysis, and interpretation of data; in the writing of the report; or in the decision to submit the report for publication.

\section{References}

1. Bates DW, Cullen DJ, Laird NM, Petersen LA, Small SD, Servi D, et al. Incidence of adverse drug events and potential adverse drug events. Implications for prevention. ADE Prevention Study Group. J Am Med Assoc 1995;274:29-34.

2. Morimoto T, Gandhi TH, Seger AC, Hsieh TC, Bates DW. Adverse drug events and medication errors: detection and classification methods. Qual Saf Health Care 2004;13:306-14.

3. Al Hamid A, Ghaleb M, Aljadhey H, Aslanpour Z. A systematic review of hospitalization resulting from medicine-related problems in adult patients. Br J Clin Pharmacol 2014;78:202-17.

4. Hilmer SN. ADME-tox issues for the elderly. Expert Opin Drug Metab Toxicol 2008;4:1321-31.

5. Caldwell J, Gardner I, Swales N. An introduction to drug disposition: the basic principles of absorption, distribution, metabolism, and excretion. Toxicol Pathol 1995;23:102-14.

6. Roberts DJ, Hall RI. Drug absorption, distribution, metabolism and excretion considerations in critically ill adults. Expert Opin Drug Metab Toxicol 2013;9:1067-84.

7. Morimoto T, Sakuma M, Matsui K, Kuramoto N, Toshiro J, Murakami J, et al. Incidence of adverse drug events and 
medication errors in Japan: the JADE study. J Gen Intern Med 2011;26:148-53.

8. Committee of Experts on Management of Safety and Quality in Health Care (SP-SQS). Expert Group on Safe Medication Practice. Glossary of term related to patient and medication safety. Available at: http://www.who.int/patientsafety/highlights/ COE_patient_and_medication_safety_gl.pdf. Accessed: 16 Nov 2017.

9. Charlson ME, Pompei P, Ales KL, MacKenzie CR. A new method of classifying prognostic comorbidity in longitudinal studies: development and validation. J Chronic Dis 1987;40:373-83.

10. Evidence-based Practice Guideline for the Treatment of CKD 2013. Available at: http://www.jsn.or.jp/guideline/pdf/CKD_evidence2013/all.pdf.

11. PAB/SD Notification No. 80 issued by the Director of Safety Division, Pharmaceutical Affairs Bureau, Ministry of Health and Welfare, dated June 29, 1992. Available at: http://www.mhlw. go.jp/shingi/2005/10/dl/s1006-4f2.pdf [in Japanese].

12. Research Priority Setting Working Group of the WHO World Alliance for Patient Safety (Allegranzi B, Angood P, Bhutta Z, Davis P, Grandt D, Hamid M, Insua J, Kaitiritimba R, Khamassi S, Madiba T, Morimoto T, Noble D, Norton P, Pang TE, Sidorchuk R, Supachutikul A, Thomas E). Summary of the evidence on patient safety: implications for research. World Health Organization. 2008.

13. Avery A, Barber N, Ghaleb M, Franklin BD, Armstrong S, Crowe $S$, et al. Investigating the prevalence and causes of prescribing errors in general practice: the PRACtICe study. London: General Medical Council, 2012.

14. Medication Errors by The Technical Series on Safer Primary Care in WHO. Available at: http://www.who.int/patientsafety/topics/ primary-care/technical_series/en/.

15. Field TS, Gurwitz JH, Avorn J, McCormick D, Jain S, Eckler M, et al. Risk factors for adverse drug events among nursing home resistants. Arch Intern Med 2001;161:1629-34.

16. Bianchetti G, Graziani G, Brancaccio D, Morganti A, Leonetti G, Manfrin M, et al. Pharmacokinetics and effects of propranolol in terminal uraemic patients and in patients undergoing regular dialysis treatment. Clin Pharmacokinet 1976;1:373-84.

17. Maher JF. Pharmacological considerations. In: Maher JF, editor. Replacement of renal function by dialysis. Boston: Kluwer Academic Publishers, 1989.

18. Gambertoglio JG. Pharmacokietic basis for drug treatment. In: Benet LZ, Massoud N, Gambertoglio JG, editors. New York: Raven Press, 1984.
19. Haughey DB, Krafy CJ, Matzke GR, Keane WF, Halstenson CE. Protein binding of disopyramide and elevated alpha-1-acid glycoprotein concentrations in serum obtained from dialysis patients and renal transplant recipients. Am J Nephrol 1985;5:35-9.

20. Lima JJ. Disopyramide. In: Evans WE, Schentag JJ, Jusco WJ, editors. Applied pharmacokinetics. 2nd ed. WA: Applied Therapeutics, Inc., 1987.

21. Belpaire FM, Van de Velde EJ, Fraeyman NH, Bogaert MG, Lameire N. Influence of continuous ambulatory peritoneal dialysis on serum alpha 1-acid glycoprotein concentration and drug binding. Eur J Clin Pharmacol 1988;35:339-43.

22. Lam YW, Banerji S, Hatfield C, Talbert RL. Principal of drug administration in renal insufficiency. Clin Pharmacokinet 1997;32:30-57.

23. Schmucker DL. Age-related changes in liver structure and function: implication for disease? Exp Gerontol 2005;40:650-9.

24. Le Couteur DG, Warren A, Cogger VC, Smedsrød B, Sørensen KK, De Cabo R, et al. Old age and the hepatic sinusoid. Anat Rec 2008;291:672-83.

25. Denic A, Glassock RJ, Rule AD. Structural and functional changes with the aging kidney. Adv Chronic Kidney Dis 2016;23:19-28.

26. Budnitz DS, Lovegrove MC, Shehab N, Richards CL. Emergency hospitalizations for adverse drug events in older Americans. $\mathrm{N}$ Engl J Med 2011;365:2002-12.

27. Choudhury D, Ahmed Z. Drug-associated renal dysfunction and injury. Nat Clin Pract Nephrol 2006;2:80-91.

28. Markowitz GS, Bomback AS, Perazella MA. Drug-induced glomerular disease: direct cellular injury. Clin J Am Soc Nephrol 2015;10:1291-9.

29. Izzedine H, Perazella MA. Thrombotic microangiopathy, cancer, and cancer drugs. Am J Kidney Dis 2015;66:857-68.

30. Gökmen MR, Cosyns JP, Arlt VM, Stiborová M, Phillips DH, Schmeiser $\mathrm{HH}$, et al. The epidemiology, diagnosis, and management of aristolochic acid nephropathy: a narrative review. Ann Intern Med 2014;158:469-77.

31. Yuan L, Kaplowitz N. Mechanism of drug-induced liver injury. Clin Liver Dis 2013;17:507-18.

32. Dreischulte T, Donnan P, Grant A, Hapca A, McCowan C, Guthrie B. Safer prescribing - a trial education, informatics, and financial incentives. N Engl J Med 2016;374:1053-64.

33. Pugh RN, Murray-Lyon IM, Dawson JL, Pietroni MC, Williams R. Transection of the oesophagus for bleeding oesophageal varice. Br J Surg 1973;60:646-9. 